\title{
Two Remarks on Extremal Equilibrium States
}

\author{
Hans-Otto Georgii \\ Mathematisches Institut der Universität Erlangen-Nürnberg, \\ D-852 Erlangen, Bismarckstr. 1/2/2, Federal Republic of Germany
}

Received February 20, 1973

\begin{abstract}
First it is shown that each extremal equilibrium state is representable as limit of Gibbs states in finite volumes, and that an analogous statement holds for extremal invariant equilibrium states. Secondly we prove that for negative pair interactions only one equilibrium state exists which minimizes (resp. maximizes) the particle density, but that in general there are more than two extremal invariant equilibrium states with the same particle density. In this context, periodic interactions are studied.
\end{abstract}

\section{Notations ${ }^{1}$}

Let us consider the $v$-dimensional cubic lattice $T=\mathbb{Z}^{v}(v \in \mathbb{N})$ and the set $\mathfrak{B}$ of all non-void subsets $V$ of $T$ with finite cardinality $|V|$. Denote by $\bar{V}$ the complement of $V$. Given any $V \subset T$, we consider the set $C_{V}=\{0,1\}^{V}$ of all configurations of particles in $V$; in particular, we set $C=C_{T}$. Further on we shall use the projections $\pi_{V}^{W}: C_{W} \rightarrow C_{V}(V \subset W \subset T)$ and $\pi_{V}=\pi_{V}^{T}$.

We assume that the particles are subjected to a pairwise interaction which is described by a function $U$ in

$$
\mathfrak{U}=\left\{U \in \mathbb{R}^{T}: U(0)=0, U(t)=U(-t)(t \in T), \sum_{t \in T}|U(t)|<\infty\right\} .
$$

Given in addition a "chemical potential" $\mu \in \mathbb{R}$, for all $V \in \mathfrak{B}$ and $\bar{c} \in C_{\bar{V}}$ the Gibbs Distribution $q_{V / \bar{c}}=q_{V / \bar{c}}^{u, U}$ on $C_{V}$ under the condition $\bar{c}$ is defined by

$$
\begin{gathered}
U_{V / \bar{c}}(c)=-\mu \sum_{t \in V} c_{t}+\frac{1}{2} \sum_{s, t \in V} c_{s} c_{t} U(s-t) \\
+\sum_{s \in V, t \in \bar{V}} c_{s} \bar{c}_{t} U(s-t) \\
Z_{V / \bar{c}}=\sum_{c \in C_{V}} \exp \left[-U_{V / \bar{c}}(c)\right], q_{V / \bar{c}}(c)=Z_{V / \bar{c}}{ }^{-1} \exp \left[-U_{V / \bar{c}}(c)\right],
\end{gathered}
$$

where $c$ is any configuration in $V$.

${ }^{1}$ For a proof of the interspersed facts, see [3], e.g. 
We denote by $\mathscr{F}$ the $\sigma$-algebra on $C$ generated by the cylindric sets, and for $V \subset T$ by $\mathscr{F}_{V}$ the $\sigma$-algebra generated by the cylindric sets which depend only on the lattice points in $V$. Then the probability measures ("states") on $\mathscr{F}$ form a convex and weakly compact set $\not \mathscr{F}$. For each state $P$, we write $P_{V}$ instead of $\pi_{V}(P)(V \subset T)$. Furthermore, for any $t \in T$ consider the corresponding shift $S_{t}$ on $C$, defined by $\left(S_{t} c\right)_{s}=c_{s+t}(s \in T)$. The states which are invariant under the transformation group $\left(S_{t}\right)_{t \in T}$, form a convex and weakly compact set $p_{0} \mathscr{F}$.

Following Dobrushin and Lanford/Ruelle, we call equilibrium state for the parameters $\mu \in \mathbb{R}$ and $U \in \mathfrak{U}$ each state $P$ with the property that for all $V \in \mathfrak{B}$ and $a \in C_{V}, q_{\bar{V} / \pi_{\bar{V}}}^{\mu, U}(a)$ is a version of the conditional probability $P\left(\pi_{V}=a / \mathscr{F}_{\bar{V}}\right)$. We write $\mathfrak{G}=\mathfrak{G}(\mu, U)$ for the set of equilibrium states and $\mathfrak{b}_{0}=\mathfrak{b}_{0}(\mu, U)=\mathfrak{b}_{\mathfrak{b}} \cap \not_{0} \mathscr{F}$ for the set of shift invariant equilibrium states. $\mathfrak{G}$ and $\mathfrak{W}_{0}$ are nonempty, convex and weakly compact. Let ex $\mathfrak{G}$ and ex $\mathfrak{S}_{0}$ be the set of the extremal points of $\left(\mathfrak{5}\right.$ and $\mathfrak{G}_{0}$, respectively. Each $P \in$ ex $\mathfrak{F}_{0}$ is called a pure phase. With the notations $\mathscr{F}_{\infty}=\bigcap_{V \in \mathfrak{B}} \mathscr{F}_{\bar{V}}$ and $\mathscr{J}=\left\{A \in \mathscr{F}: A=S_{t} A(t \in T)\right\}$ we have the following characterizations:

$$
\begin{aligned}
& P \in \mathfrak{G} \text { is extremal in } \mathfrak{5} \text {, iff } P(A) \in\{0,1\} \text { for all } A \in \mathscr{F}_{\infty} \\
& P \in \mathfrak{G}_{0} \text { is extremal in } \mathfrak{G}_{0} \text {, iff } P(A) \in\{0,1\} \text { for all } A \in \mathscr{J},
\end{aligned}
$$

i.e., the pure phases are exactly the ergodic equilibrium states.

Furthermore, if $U \in \mathfrak{U}^{-}=\{U \in \mathfrak{U}: U \leqq 0\}$, then Ruelle has proved the existence of two states $P^{-}, P^{+} \in \mathfrak{G}_{0}$ with the property

$$
P_{X}^{-}(1) \leqq P_{X}(1) \leqq P_{X}^{+}(1) \quad \text { for all } \quad X \in \mathfrak{B} \text { and all } P \in \mathfrak{G},
$$

which implies the extremality of $P^{-}$and $P^{+}$in $\mathfrak{5}$ and in $\mathfrak{G}_{0}$. Given $X \subset V \in \mathfrak{B}$ and $a \in C_{X}$, one considers the functions

$$
I_{X, a}(c)= \begin{cases}1 & \text { if } \pi_{X}^{V}(c)=a \\ 0 & \text { otherwise }\end{cases}
$$

and the expectation $r_{V / \bar{c}}(X)=E_{V / \bar{c}}\left(I_{X, 1}\right)$ of $I_{X, 1}$ with respect to the measure $q_{V / \bar{c}} . r_{V / \bar{c}}$ is called the correlation function of $q_{V / \bar{c}}$. Then for all $X$

$$
P_{X}^{-}(1)=\lim _{V\urcorner T} r_{V / 0}(X), \quad P_{X}^{+}(1)=\lim _{V \succ T} r_{V / 1}(X) .
$$

Finally, we set $\varrho_{-}=\varrho_{-}(\mu, U)=P_{\{0\}}^{-}(1)$ and $\varrho_{+}=\varrho_{+}(\mu, U)=P_{\{0\}}^{+}(1)$. Note that for $P \in p_{0} \mathscr{F}$ the number $P_{\{0\}}(1)$ can be interpreted as mean particle density for $P$. 


\section{Approximation of Extremal Equilibrium States}

Consider the set $\mathfrak{G}_{\infty}$ of all states $P$ for which there is an isotone sequence $\left(V_{k}\right)_{k \in \mathbb{N}}$ in $\mathfrak{B}$ tending to $T$, and configurations $\bar{c}_{k} \in C_{\bar{V}_{k}}$ such that for all $X \in \mathfrak{B}$,

$$
P_{X}(1)=\lim _{k \rightarrow \infty} r_{V_{k} / \bar{c}_{k}}(X) \text {. }
$$

Then $\left(\mathfrak{b}\right.$ and the closed convex hull of $\mathfrak{b}_{\infty}$ coincide (cf. [3]). It is therefore natural to ask whether the relation ex $\mathfrak{b}_{\infty} \subset \mathfrak{b}_{\infty}$ is true. The answer is: Yes.

Let $X \in \mathfrak{B}$ be any finite set and $\mathfrak{X}=\{V \in \mathfrak{B}: V \supset X\}$. Given any function $f$ on $C_{X}$, we put

$$
f_{V}(c)=E_{V / \pi \bar{V}(c)}\left(f \circ \pi_{X}^{V}\right) \quad(V \in \mathfrak{X}, c \in C) .
$$

For equilibrium states $P \in \mathfrak{G}$ we have by definition

$$
f_{V}=E\left(f \circ \pi_{X} / \mathscr{F}_{\bar{V}}\right) \quad P \text {-a.s. }(V \in \mathfrak{X}) .
$$

This shows the validity of

Proposition 2.1. For each $P \in \mathfrak{G}$ and $X \in \mathfrak{B}$, the family $\left(f_{V}\right)_{V \in \mathfrak{X}}$ is a (backward-) martingale with respect to the family of $\sigma$-algebras $\left(\mathscr{F}_{\bar{V}}\right)_{V \in \mathfrak{X}}$ and the measure $P$.

It is an interesting consequence of this proposition that each equilibrium state $P$ depends only on its restriction on $\mathscr{F}_{\infty}$. By the martingale convergence theorem (cf. [4], p. 120), if $V$ runs through an increasing sequence in $\mathfrak{B}$ to $T$, the sequence $r_{V / \pi \bar{V}}(X)$ converges $P$-a.e. and in $L^{1}(P)$ norm to a $\mathscr{F}_{\infty}$-measurable function $g_{X}$, and we have

$$
P_{X}(1)=\int g_{X} d P=\left.\int g_{X} d P\right|_{\mathscr{F}_{\infty}} .
$$

Now the remark follows from the fact that $P$ is completely determined by the system $\left(P_{X}(1)\right)_{X \in \mathfrak{B}}$.

Theorem 2.2. Let $P \in \operatorname{ex}(\mathfrak{5}$ be an extremal equilibrium state and $\left(V_{k}\right)_{k \in \mathbb{N}}$ any isotone sequence in $\mathfrak{B}$ tending to $T$. Then

$$
P\left\{P_{X}(1)=\lim _{k \rightarrow \infty} r_{V_{k} / \pi \bar{v}_{k}}(X) \text { for all } X \in \mathfrak{B}\right\}=1 .
$$

In particular, the inclusion ex $\mathfrak{G}_{C} \mathfrak{G}_{\infty}$ is true.

Proof. Using the notations and results of the preceding remark, we obtain from (1.2) that $g_{X}=P_{X}(1) P$-a.s. Hence

$$
P\left\{r_{V_{k} / \pi \bar{v}_{k}}(X) \rightarrow P_{X}(1)(k \rightarrow \infty)\right\}=1
$$

for any $X \in \mathfrak{B}$, and the theorem follows, because $\mathfrak{B}$ is countable.

Now we study the analogous problem for extremal invariant equilibrium states. One introduces the averaged correlation functions

$$
\bar{r}_{V / \bar{c}}(X)=|V|^{-1} \sum_{t \in T: X+t \subset V} r_{V / \bar{c}}(X+t) \quad\left(X \subset V \in \mathfrak{B}, \bar{c} \in C_{\bar{V}}\right)
$$


and the set $\mathfrak{G}_{0, \infty}$ of all invariant states $P \in \not_{0} \mathscr{F}$ with the property that there is an increasing sequence $\left(V_{k}\right)_{k \in \mathbb{N}}$ in $\mathfrak{B}$ tending to $T$, and configurations $\bar{c}_{k} \in C_{\bar{V}_{k}}$ such that for all $X \in \mathfrak{B}$

$$
P_{X}(1)=\lim _{k \rightarrow \infty} \bar{r}_{V_{k} / \bar{c}_{k}}(X) \text {. }
$$

We show that ex $\mathfrak{5}_{0} \subset \mathfrak{5}_{0, \infty}$.

Proposition 2.3. ${ }^{2}$ Let $(\Omega, \mathscr{B}, m)$ be any probability space, $\left(\mathscr{B}_{n}\right)_{n \in \mathbb{N}}$ a decreasing family of $\sigma$-subalgebras of $\mathscr{B}, \mathscr{B}_{\infty}=\bigcap_{n \in \mathbb{N}} \mathscr{B}_{n}$ and $\left(f_{n}\right)_{n \in \mathbb{N}}$ any sequence of random variables in $L^{1}(m)$. If $\left|f_{n}\right| \leqq g$ for some $g \in L^{1}(m)$ and $f_{n} \rightarrow f$ a.s., then

$$
E\left(f_{n} / \mathscr{B}_{n}\right) \rightarrow E\left(f / \mathscr{B}_{\infty}\right) \text { a.s. and in } L^{1} \text {-norm, }
$$

if $n$ tends to infinity.

Proof. It is enough to verify the "Fatou inequality"

$$
E\left(\liminf _{n \rightarrow \infty} f_{n} / \mathscr{B}_{\infty}\right) \leqq \liminf _{n \rightarrow \infty} E\left(f_{n} / \mathscr{B}_{\infty}\right)
$$

for any sequence $\left(f_{n}\right)_{n \in \mathbb{N}}$ in $L^{1}$ such that $f_{n} \geqq g$ for some $g \in L^{1}$. Consider the random variables $g_{k}=\inf _{n \geqq k} f_{n}(k \in \mathbb{N})$. The inequality $f_{n} \geqq g_{k} \geqq g(n \geqq k)$ implies that $g_{k} \in L^{1}$. Thus the martingale convergence theorem applies showing that

$$
\begin{aligned}
E\left(g_{k} / \mathscr{B}_{\infty}\right) & =\lim _{n \rightarrow \infty} E\left(g_{k} / \mathscr{B}_{n}\right) \\
& \leqq \liminf _{n \rightarrow \infty} E\left(f_{n} / \mathscr{B}_{n}\right) .
\end{aligned}
$$

Now $g_{k} \gg \lim \inf f_{n}$ if $k \rtimes \infty$. By the monotone convergence theorem (cf. [4], p. 50), (2.2) follows.

Corollary 2.4. For any $P \in \mathfrak{G}_{0}$, any $X \in \mathfrak{B}$ and any isotone sequence $\left(V_{k}\right)_{k \in \mathbb{N}}$ of cubes in $\mathfrak{B}$ tending to $T$, the sequence

$$
\left(\bar{r}_{V_{k} / \pi \bar{v}_{k}}(X)\right)_{k \in \mathbb{N}}
$$

converges $P$-a.s. and in $L^{1}(P)$-norm to $P\left(\pi_{X}=1 / \mathscr{J}\right)$.

Proof. By (2.1) for any $P \in \mathfrak{G}_{0}$ and $V \in \mathfrak{X}$ we have

$$
\bar{r}_{V / \pi \bar{V}}(X)=E\left(|V|^{-1} \sum_{X+t \subset V} 1_{\left\{\pi_{X}=1\right\}} \circ S_{-t} / \mathscr{F}_{\bar{V}}\right) \text { a.s. }
$$

The $v$-dimensional ergodic theorem (cf. [5]) implies that

$$
\left|V_{k}\right|^{-1} \sum_{X+t \subset V_{k}} 1_{\left\{\pi_{X}=1\right\}}{ }^{\circ} S_{-t} \rightarrow P\left(\pi_{X}=1 / \mathscr{J}\right) \text { a.s. }
$$

Thus Proposition 2.3 applies, asserting that

$$
\bar{r}_{V_{k} / \bar{V}_{k}}(X) \rightarrow E\left(P\left(\pi_{X}=1 / \mathscr{J}\right) / \mathscr{F}_{\infty}\right)=P\left(\pi_{X}=1 / \mathscr{J}\right)
$$

2 This statement already has been remarked by G. A. Hunt (Les Martingales, Seminaire d'Orsay 1963, p. 58). 
a.s. and in $L^{1}$-norm. For the last equality we have used Proposition 3.6 below.

Using (1.2) and the countability of $\mathfrak{B}$, we get from Corollary 2.4

Theorem 2.5. If $P \in \operatorname{ex} \mathfrak{b}_{0}$, then for any increasing sequence $\left(V_{k}\right)_{k \in \mathbb{N}}$ of cubes in $\mathfrak{B}$ tending to $T$ we have

$$
P\left\{P_{X}(1)=\lim _{k \rightarrow \infty} \bar{r}_{V_{k} / \pi \bar{v}_{k}}(X) \text { for all } X \in \mathfrak{B}\right\}=1 .
$$

In particular, ex $\mathfrak{G}_{0} \subset \mathfrak{G}_{0, \infty}$.

Finally it should be remarked that the results of this section can be generalized without difficulties to classical systems with many-body interactions (which are subjected to the usual summability condition).

\section{Pure Phases for Attractive Interactions}

For $U \in \mathfrak{U}^{-}$the theorem of Lee and Yang (cf. [3], e.g.) asserts that a phase transition (i.e. $\varrho_{-}<\varrho_{+}$) can occur only for the chemical potential

$$
\hat{\mu}(U)=\frac{1}{2} \sum_{s \in T} U(s) \text {. }
$$

It is natural to wonder whether in this situation at most two pure phases exist which are limits of the uniquely determined (cf. [3]) pure phases for $\mu<\hat{\mu}(U)$ and $\mu>\hat{\mu}(U)$, respectively. The equilibrium states $P^{-}$and $P^{+}$ are natural candidates for these two pure phases. For the Ising interaction

$$
\mathfrak{J}_{\beta}(t)=\left\{\begin{aligned}
-\beta & \text { if } t= \pm e \text { for some unit vector } e \\
0 & \text { otherwise }
\end{aligned}\right.
$$

at low enough temperature $\beta^{-1}>0$ the answer to the question above is affirmative, as proved by Gallavotti and Miracle-Solé (cf. [3]). We show that $P^{-}$and $P^{+}$are the unique equilibrium states with particle density $\varrho_{-}$and $\varrho_{+}$, respectively, but that for a big class of negative interactions there exist more than these two pure phases.

Lemma 3.1. Given any $U \in \mathfrak{U}^{-}$and $\mu \in \mathbb{R}$, let $P, Q \in \mathfrak{G}(\mu, U)$ have the properties $P_{\{t\}}(1)=\varrho_{-}(\mu, U), Q_{\{t\}}(1)=\varrho_{+}(\mu, U)$ for all $t \in T$. Then for all $X \in \mathfrak{B}$ and $s \in X$,

$$
P_{X}^{-}\left(1_{Y}\right) \leqq P_{X}\left(1_{Y}\right), \quad Q_{X}\left(1_{Y}\right) \leqq P_{X}^{+}\left(1_{Y}\right),
$$

where $Y=X \backslash\{s\}$ and

$$
1_{Y}(t)=\left\{\begin{array}{lll}
1 & \text { for } & t \in Y \\
0 & \text { for } & t=s
\end{array} .\right.
$$

Proof. Choose any $V \in \mathfrak{X}$ and $\bar{c} \in C_{\bar{V}}$. Define

$$
h(c)=\exp \left[-\sum_{t \in V \backslash\{s\}, t^{\prime} \in \bar{V}} c_{t} \bar{c}_{t^{\prime}} U\left(t^{\prime}-t\right)\right]\left(c \in C_{V \backslash\{s\}}\right) .
$$


Then by a theorem of Fortuin, Ginibre and Kasteleyn (cf. [3]) we have

Hence

$$
\begin{aligned}
\frac{Z_{V / \bar{c}} E_{V / \bar{c}}\left(I_{Y, 1} I_{\{s\}, 0}\right)}{Z_{V / 0} E_{V / 0}\left(I_{Y, 1} I_{\{s\}, 0}\right)} & =\frac{E_{V \backslash\{s\} / 0}\left(I_{Y, 1} h\right)}{E_{V \backslash\{s\} / 0}\left(I_{Y, 1}\right)} \\
& \geqq E_{V \backslash\{s\} / 0}(h)=\frac{Z_{V / \bar{c}} E_{V / \bar{c}}\left(I_{\{s\}, 0}\right)}{Z_{V / 0} E_{V / 0}\left(I_{\{s\}, 0}\right)} .
\end{aligned}
$$

$$
\begin{aligned}
\frac{P_{X}\left(1_{Y}\right)}{P_{X}^{-}\left(1_{Y}\right)} & =\lim _{V \nmid T} \int \frac{E_{V / \bar{c}}\left(I_{Y, 1} I_{\{s\}, 0}\right)}{E_{V / 0}\left(I_{Y, 1} I_{\{s\}, 0}\right)} P_{\bar{V}}(d \vec{c}) \\
& \geqq \lim _{V \backslash T} \int \frac{E_{V / \bar{c}}\left(I_{\{s\}, 0}\right)}{E_{V / 0}\left(I_{\{s\}, 0}\right)} P_{\bar{V}}(d \vec{c})=\frac{P_{\{s\}}(0)}{P_{\{s\}}^{-}(0)}=1 .
\end{aligned}
$$

The second inequality can be proved similarly.

If we take into account that by (1.4) $P_{\{t\}}(1) \in\left[\varrho_{-}, \varrho_{+}\right]$for all $P \in \mathfrak{G}$ and $t \in T$, the following theorem has some interesting consequences. First it illustrates the kind of extremality of $P^{-}$and $P^{+}$in $\mathfrak{G}$ and in $\mathfrak{5}_{0}$. Furthermore it shows that for $\varrho_{-}=\varrho_{+}$("no phase transition of first order") only one equilibrium state exists. In particular, for $U \in \mathfrak{U}^{-}$the "absence of a symmetry breakdown" (i.e. $\mathfrak{G}_{0} \subset$ ex $(\mathfrak{5})$ implies the uniqueness of the equilibrium state.

Theorem 3.2. For all $U \in \mathfrak{U}^{-}$and $\mu \in \mathbb{R}$,

and

$$
\left\{P \in \mathfrak{G}(\mu, U): P_{\{t\}}(1)=\varrho_{-}(\mu, U) \text { for all } t \in T\right\}=\left\{P^{-}\right\}
$$

$$
\left\{Q \in \mathfrak{G}(\mu, U): Q_{\{t}(1)=\varrho_{+}(\mu, U) \text { for all } t \in T\right\}=\left\{P^{+}\right\} .
$$

Proof. Suppose that $P \in \mathfrak{G}$ has the property $P_{\{t\}}(1)=\varrho_{-}(t \in T)$. Then we have the identity

$$
P_{\{t\}}(1)=P_{\{t\}}^{-}(1) \quad(t \in T) .
$$

If for some $X \in \mathfrak{B} P_{X}(1) \neq P_{X}^{-}(1)$, then by (1.4) and Lemma 3.1

$$
P_{Y}(1)-P_{Y}^{-}(1)=P_{X}(1)-P_{X}^{-}(1)+P_{X}\left(1_{Y}\right)-P_{X}^{-}\left(1_{Y}\right)>0 \text {. }
$$

By iteration, we get a contradiction to (3.1). Do the same for the second assertion.

Now let $T=T^{\prime} \cup T^{\prime \prime}$ be any partition of $T$ in two disjoint subsets $T^{\prime}$ and $T^{\prime \prime}$ of $T$. Given any configuration $c$ in some subset $V$ of $T$, the configuration $\varphi c$ in $V$ let be defined by

$$
(\varphi c)_{t}= \begin{cases}1-c_{t} & \text { if } t \in V^{\prime}=V \cap T^{\prime} \\ c_{t} & \text { if } t \in V^{\prime \prime}=V \cap T^{\prime \prime} .\end{cases}
$$

Lemma 3.3. Suppose that $U \in \mathfrak{U}^{\prime}=\left\{U \in \mathfrak{U}: U(s-t)=0\left(s \in T^{\prime}, t \in T^{\prime \prime}\right)\right\}$ and $\mu=\hat{\mu}(U)$. Then the equality

holds.

$$
q_{V / \bar{c}}(c)=q_{V / \varphi \bar{c}}(\varphi c) \quad\left(c \in C_{V}, \bar{c} \in C_{\bar{V}}, V \in \mathfrak{B}\right)
$$


Proof. Direct computation yields

$$
U_{V / \bar{c}}(c)-U_{V / \varphi \bar{c}}(\varphi c)=\sum_{s \in V^{\prime}, t \in \bar{V}^{\prime}}\left[\bar{c}_{t}-\frac{1}{2}\right] U(s-t) .
$$

As the righthand side is independent of $c$, the lemma follows.

Lemma 3.4. Suppose that $U \in \mathfrak{U}^{-} \cap \mathfrak{U}^{\prime}$ and $\mu=\hat{\mu}(U)$. Then for all $X \subset V \subset W \in \mathfrak{B}$ and $\bar{c} \in C_{\bar{V}}$

and

$$
\begin{aligned}
& E_{V / \varphi 0}\left(I_{X, \varphi 1}\right) \leqq E_{V / c}\left(I_{X, \varphi 1}\right) \leqq E_{V / \varphi 1}\left(I_{X, \varphi 1}\right) \\
& E_{V / \varphi 0}\left(I_{X, \varphi 1}\right) \leqq E_{W / \varphi 0}\left(I_{X, \varphi 1}\right) .
\end{aligned}
$$

Proof. By the lemma before,

$$
E_{V / \varphi \bar{c}}\left(I_{X, \varphi 1}\right)=E_{V / \bar{c}}\left(I_{X, 1}\right) .
$$

Therefore the assertion is a consequence of the similar properties of the correlation functions $r_{V / \bar{c}}(X)$ (cf. [3], Lemma 10.2).

Lemma 3.5. Each state $P \in \not{F F}$ is uniquely determined by the system $\left(P_{X}(\varphi 1)\right)_{X \in \mathfrak{B}}$.

Proof. By the inclusion-exclusion formula, clearly for all $V \in \mathfrak{B}$

$$
P_{V}(1)=\sum_{V^{\prime \prime} \subset X \subset V}(-1)^{\left|X \backslash V^{\prime \prime}\right|} P_{X}(\varphi 1) .
$$

But $P$ is uniquely determined by the system $\left(P_{V}(1)\right)_{V \in \mathfrak{B}}$ (cf. [3], e.g.).

Finally we shall need the following fact which in the 1-dimensional case is well-known.

Proposition 3.6. For all $P \in \mu_{0} \mathscr{F}, \mathscr{J} \subset \mathscr{F}_{\infty} P$-a.s.

Proof. ${ }^{3}$ It is sufficient to show that each invariant function $f \in L^{1}(P)$ coincides a.s. with its conditional expectation $E\left(f / \mathscr{F}_{\infty}\right)$. Let $\|\cdot\|$ denote the $L^{1}(P)$-norm. Then for $V, W \in \mathfrak{B}, W \subset \bar{V}$,

$$
\begin{aligned}
\left\|f-E\left(f / \mathscr{F}_{\infty}\right)\right\| \leqq & \left\|f-E\left(f / \mathscr{F}_{W}\right)\right\| \\
& +\left\|E\left(f / \mathscr{F}_{W}\right)-E\left(f / \mathscr{F}_{\bar{V}}\right)\right\|+\left\|E\left(f / \mathscr{F}_{\bar{V}}\right)-E\left(f / \mathscr{F}_{\infty}\right)\right\| .
\end{aligned}
$$

If $V$ runs through an increasing sequence in $\mathfrak{B}$ to $T$, by the convergence theorem for backward martingales the third summand becomes small. By Jensen's inequality ([4], p. 50) the second term is majorized by the first one. Using the invariance of $f$ and $P$, for all $t \in T$ we obtain

$$
\left\|f-E\left(f / \mathscr{F}_{W}\right)\right\|=\left\|f-E\left(f / \mathscr{F}_{W+t}\right)\right\| .
$$

${ }^{3}$ This proof is due to H. Föllmer; it simulates Meyer's proof of the Hewitt-Savage 0-1 law (cf. [4], p. 190). 
Therefore, we can forget the condition $W \subset \bar{V}$ and let $W$ run through an isotone sequence in $\mathfrak{B}$ to $T$, and by the forward martingale convergence theorem (cf. [4], p. 117) the proposition follows.

Suppose that $U \in \mathfrak{U}$ is given. We call $s, t \in T U$-equivalent if there is a finite sequence $t_{0}, \ldots, t_{n}$ in $T$ such that $t_{0}=s, t_{n}=t$ and $U\left(t_{k}-t_{k-1}\right) \neq 0$ $(1 \leqq k \leqq n)$. For any $s \in T$ denote by $T_{s}$ the set of all points in $T$ which are $U$-equivalent to $s$.

Definition. We say that $U \in \mathfrak{U}$ is periodic if $U \neq 0$ and if there is a parallelepiped $\mathscr{W}_{U} \in \mathfrak{B}$ of cardinality greater than one with the following properties:

(a) $T_{u} \cap T_{v}=\emptyset\left(u, v \in \mathscr{W}_{U}, u \neq v\right)$

(b) $T_{u}+\left(n_{1}, 0, \ldots, 0\right)=\cdots=T_{u}+\left(0, \ldots, 0, n_{v}\right)=T_{u}\left(u \in \mathscr{W}_{U}\right)$, where $n_{j} \in \mathbb{N}$ denotes the vertex length of $\mathscr{W}_{U}$ in direction $j(1 \leqq j \leqq v)$.

For most of the following it would be enough to require $\bigcup_{u \in W_{U}} T_{u}=T$ instead of (b).

If $U$ is some periodic interaction, let $\mathscr{M}_{U}$ be the set of all subsets of $\mathscr{W}_{U}$. For any $M \in \mathscr{M}_{U}$ and $t \in T, M+t=\left(T_{M}+t\right) \cap \mathscr{W}_{U}$ denotes the "modulo $\mathscr{W}_{U}$ " shifted set and $\bar{M}=\mathscr{W}_{U} \backslash M$ the complement of $M$. Given any $M \in \mathscr{M}_{U}$, consider the lattice

$$
T_{M}=\bigcup_{u \in M} T_{u}
$$

and the map $\varphi_{M}$ defined as $\varphi$ for the partition $T^{\prime}=T_{M}, T^{\prime \prime}=T_{\bar{M}}$.

Theorem 3.7. Let $U \in \mathfrak{U}^{-}$be any periodic interaction and $\mu=\hat{\mu}(U)$. Then the equilibrium states $P^{M}\left(M \in \mathscr{M}_{U}\right)$ defined by

$$
P_{X}^{M}(1)=\lim _{V \uparrow T} r_{V / \varphi_{M} 0}(X) \quad(X \in \mathfrak{B})
$$

are extremal in $\mathfrak{5}(\mu, U)$. For any $M \in \mathscr{M}_{U}$ the following statements are true:

(a) $P^{\theta}=P^{-}, P^{\mathscr{W}_{U}}=P^{+}$

(b) $P_{X}^{M}\left(\varphi_{M} 1\right) \leqq P_{X}\left(\varphi_{M} 1\right) \leqq P_{X}^{\bar{M}}\left(\varphi_{M} 1\right)(P \in \mathfrak{G}(\mu, U), X \in \mathfrak{B})$

(c) For all $X \in \mathfrak{B}$ such that $\left|X \cap T_{u}\right| \leqq 1\left(u \in \mathscr{W}_{U}\right)$, $P_{X}^{M}(1)=\varrho_{+}^{\left|X \cap T_{M}\right|} \varrho_{-}^{\left|X \cap T_{\bar{M}}\right|}$

(d) $P^{M}\left(S_{t} A\right)=P^{M-t}(A)(A \in \mathscr{F}, t \in T)$

(e) $P^{M}(1-A)=P^{\bar{M}}(A)(A \in \mathscr{F}, 1-A=\{1-c: c \in A\})$.

Clearly the probability measures $P^{M}$ are representable as products of the measures $\left.P^{-}\right|_{u}$ and $\left.P^{+}\right|_{u}$ which are obtained by restricting (1.6) to the lattice $T_{u}\left(u \in \mathscr{W}_{U}\right)$.

Proof. The existence of the limits in (3.3) follows from Lemma 3.4, Eq. (3.2) and the correspondent equation for the measures $q_{V / \varphi_{M} 0}$. (a) is true by definition, and (b) by Lemma 3.4. By Lemma (3.5), (b) implies the extremality of the measures $P^{M}$. For the proof of (c) observe that

$$
q_{V / \bar{c}}=\prod_{u \in \mathscr{W}_{U}} q_{V \cap T_{u} / \pi_{T_{u}}(\bar{c})} .
$$


This implies that $\varrho_{-}=\lim _{V \uparrow T} r_{V \cap T_{u} / 0}(\{t\})$ and $\varrho_{+}=\lim _{V \uparrow T} r_{V \cap T_{u} / 1}(\{t\})\left(t \in T_{u}\right.$, $\left.u \in \mathscr{W}_{U}\right)$. For $X \in \mathfrak{B}$ such that $\left|X \cap T_{u}\right| \leqq 1$ we get

$$
\begin{aligned}
P_{X}^{M}(1) & =\lim _{V \ni T} \prod_{u \in \mathscr{W}_{U}: X \cap T_{u} \neq \emptyset} r_{V \cap T_{u} / \varphi_{u} 0}\left(X \cap T_{u}\right) \\
& =\varrho_{+}^{\left|X \cap T_{M}\right|} \varrho_{-}^{\left|X \cap T_{\bar{M}}\right|} .
\end{aligned}
$$

Finally it is enough to prove (d) and (e) for sets $A$ of the form $\pi_{X}{ }^{-1}(1)$ $(X \in \mathfrak{B})$. But

$$
\begin{aligned}
P_{X+t}^{M}(1) & =\lim _{V \uparrow T} r_{V-t / S_{-t}\left(\varphi_{M} 0\right)}(X) \\
& =\lim _{W \succ T} r_{W / \varphi_{M-t} 0}(X)=P_{X}^{M-t}(1),
\end{aligned}
$$

and Lemma 3.3 shows the validity of the equation

$$
E_{V / \varphi_{M} 0}\left(I_{X, 1}\right)=E_{V / 1-\varphi_{M} 0}\left(I_{X, 0}\right)
$$

which implies $P_{X}^{M}(1)=P_{X}^{\bar{M}}(0)$ and therefore (e).

We call $M, L \in \mathscr{M}_{U}$ toroidal equivalent, iff there is a $t \in T$ such that $M=L+t$. Each equivalence class is called a toroidal set. For any $M \in \mathscr{M}_{U}$ we denote by $[M]$ the toroidal set generated by $M$. Let $\mathscr{A}_{U}$ be the collection of all toroidal sets. By the Möbius inversion theorem (cf. [1], p. 70/73), its cardinality can be expressed as

$$
\left|\mathscr{A}_{U}\right|=\sum_{\substack{k_{1}\left|n_{1} \\ \ldots, \ldots \\ k_{v}\right| n_{v}}} \frac{1}{k_{1} \ldots k_{v}} \sum_{\substack{j_{1}\left|k_{1} \\ \ldots, k_{v} \\ j_{v}\right| k_{v}}} \mu\left[\left(j_{1}, \ldots, j_{v}\right),\left(k_{1}, \ldots, k_{v}\right)\right] 2^{j_{1} \ldots j_{v}},
$$

where "l" means "divisor of" and $\mu[.,$.$] is the Möbius function (cf. [1]).$ Observe that $\left|\mathscr{A}_{U}\right| \geqq\left|\mathscr{W}_{U}\right|+1$. For any $\alpha \in \mathscr{A}_{U}$, let $|\alpha|$ be the number of toroidal equivalent sets in $\alpha, k(\alpha)$ the cardinality of any $M \in \alpha$ and $\bar{\alpha}=[\bar{M}]$ for some $M \in \alpha$.

Corollary 3.8. Suppose that $U \in \mathfrak{U}^{-}$is periodic and $\mu=\hat{\mu}(U)$. Then for any toroidal set $\alpha \in \mathscr{A}_{U}$ the equilibrium state $P^{\alpha}$ defined by

$$
P^{\alpha}=|\alpha|^{-1} \sum_{M \in \alpha} P^{M}
$$

is extremal in $\mathfrak{b}_{0}(\mu, U)$ and has the following properties:

(a) $P^{[\emptyset]}=P^{\natural}=P^{-}, P^{\left[\mathscr{W}_{U}\right]}=P^{\mathscr{W}_{U}}=P^{+}$

(b) $P_{\{0\}}^{\alpha}(1)=\frac{k(\alpha)}{\left|\mathscr{W}_{U}\right|} \varrho_{+}(\mu, U)+\left[1-\frac{k(\alpha)}{\left|\mathscr{W}_{U}\right|}\right] \varrho_{-}(\mu, U)$

(c) $P^{\alpha}(1-A)=P^{\bar{\alpha}}(A) \quad(A \in \mathscr{F})$.

In particular, if $\left|\mathscr{W}_{U}\right|$ is even, then there exist pure phases which are invariant under the "Spin Flip" $c \rightarrow 1-c$. 
Proof. By statement (d) of the theorem, $P^{\alpha} \in \mathfrak{G}_{0}$. In view of (1.3) it is enough to show that $P^{\alpha}$ is ergodic. Let $A \in \mathscr{J}$ be any invariant set. Then Lemma 3.6 guarantees the existence of some $B \in \mathscr{F}_{\infty}$ such that $P^{\alpha}(A \triangle B)=0\left(\triangle\right.$ symmetric difference) and therefore $P^{M}(A \triangle B)=0$ for all $M \in \alpha$. By $(1.2) P^{M}(B)=0$ or 1 . Hence $P^{M}(A)=0$ or 1 for all $M \in \alpha$. Statement (d) of the theorem shows that the measures $P^{M}(M \in \alpha)$ coincide on $\mathscr{J}$. Thus $P^{\alpha}(A)=0$ or 1 . The properties (a)-(c) are immediate consequences of the statements (a), (c) and (e) of the theorem. If $\left|\mathscr{W}_{U}\right|$ is even, we can produce some $\alpha$ with $\alpha=\bar{\alpha}$ by dividing $\mathscr{W}_{U}$ into two congruent subsets.

Now we investigate the question whether the states $P^{M}\left(M \in \mathscr{M}_{U}\right)$ and $P^{\alpha}\left(\alpha \in \mathscr{A}_{U}\right)$ are different, respectively. If $\mu \neq \hat{\mu}(U)$ then there exists only one equilibrium state, i.e., all $P^{M}$ and $P^{\alpha}$ are identical. If $\mu=\hat{\mu}(U)$ but $\varrho_{-}=\varrho_{+}$, by Theorem 3.2 the same holds. If $\varrho_{-}<\varrho_{+}$(and there are negative periodic interactions $U$ such that $\varrho_{-}(\hat{\mu}(\beta U), \beta U)<\varrho_{+}(\hat{\mu}(\beta U), \beta U)$ for sufficiently low temperature $\beta^{-1}$, cf. [3]), then statement (c) of the theorem shows that

$$
P_{\{t\}}^{M}(1) \neq P_{\{t\}}^{L}(1) \quad\left(t \in T_{M \triangle L}, M, L \in \mathscr{M}_{U}, M \neq L\right)
$$

and therefore the measures $P^{M}\left(M \in \mathscr{M}_{U}\right)$ are pairwise different. Furthermore, statement (b) of Corollary 3.8 shows that $\left|\mathscr{W}_{U}\right|+1$ different pure phases with different particle densities exist. In order to distinguish pure phases $P^{\alpha}$ with the same particle density we need some additional investigations.

Let $\alpha, \beta \in \mathscr{A}_{U}$ be two distinct toroidal sets, $\alpha=[A], \beta=[B]$. Then there exists a discriminant set $D \in \mathscr{M}_{U}$ such that $|D(A)| \neq|D(B)|$, where we use the abbreviation

$$
D(M)=\bigcap_{u \in D}(M-u)=\left\{v \in \mathscr{W}_{U}: D+v \subset M\right\} \quad\left(M \in \mathscr{M}_{U}\right) .
$$

For instance, if $|A| \geqq|B|$,

$$
|A(A)|=|\alpha|^{-1}\left|\mathscr{W}_{U}\right|>0=|A(B)| .
$$

Choose $D \in \mathscr{M}_{U}$ such that for all $M \subset D, M \neq D,|M(A)|=|M(B)|$. Then the following lemma applies, showing that for $\varrho_{-}<\varrho_{+}$the pure phases $P^{\alpha}$ are pairwise distinct.

Lemma 3.9. Suppose that $U \in \mathfrak{U}^{-}$is periodic and $\mu=\hat{\mu}(U)$. Let $\alpha, \beta \in \mathscr{A}_{U}$ be two toroidal sets, $\alpha=[A], \beta=[B]$. Assume that $D \in \mathscr{M}_{U}$ is chosen such that $|M(A)|=|M(B)|$ for all proper subsets $M$ of $D$. Then for any $X \in \mathfrak{B}$ with the property

$$
\left|X \cap T_{u}\right|=\left\{\begin{array}{lll}
1 & \text { for } & u \in D \\
0 & \text { for } & u \in \bar{D}
\end{array}\right.
$$


the equation

holds.

$$
P_{X}^{\beta}(1)-P_{X}^{\alpha}(1)=\left|W_{U}\right|^{-1}[|D(B)|-|D(A)|]\left[\varrho_{+}-\varrho_{-}\right]^{|X|}
$$

Proof. Assume that $|D(B)|-|D(A)|=\delta \geqq 0$. For any two disjoint subsets $K, L$ of $D$ consider the sets

$$
D_{K, L}(A)=K(A) \cap L(\bar{A}),
$$

$D_{K, L}(B), D_{L}(A)=D_{D \backslash L, L}(A)$ and $D_{L}(B)$. They constitute two partitions $\left(D_{L}(A)\right)_{L \subset D}$ and $\left(D_{L}(B)\right)_{L C D}$ of $\mathscr{W}_{U}$. It is easy to show by induction on $|L|$ that

and

$$
\left|D_{K, L}(A)\right|=\left|D_{K, L}(B)\right| \quad(K \cup L \underset{\neq}{C} D)
$$

$$
(-1)^{|L|}\left[\left|D_{L}(B)\right|-\left|D_{L}(A)\right|\right]=\delta \quad(L \subset D) \text {. }
$$

The second equality guarantees the existence of some permutation $\pi$ of $\mathscr{W}_{U}$ such that

$$
\begin{aligned}
\pi\left(D_{L}(A)\right) \subset D_{L}(B) & (|L| \text { even }) \\
\pi^{-1}\left(D_{L}(B)\right) \subset D_{L}(A) & (|L| \text { odd }) .
\end{aligned}
$$

Now if $u \in D_{L}(A)\left(|L|\right.$ even) or $u \in \pi^{-1}\left(D_{L}(B)\right)(|L|$ odd $)$, then

$$
|(D+u) \cap A|=|D \backslash L|=\left|\left(D+\pi_{u}\right) \cap B\right| .
$$

Thus by assertion (c) of Theorem 3.7 and Eq. (3.5)

$$
\begin{aligned}
\left|W_{U}\right|\left[P_{X}^{\beta}(1)-P_{X}^{\alpha}(1)\right] & \\
= & \sum_{u \in W_{U}}\left[\varrho_{+}^{\left|X \cap T_{B-u}\right|} \varrho_{-}^{\left|X \cap T_{B}-u\right|}-\varrho_{+}^{\left|X \cap T_{A-u}\right|} \varrho_{-}^{\left.\mid X \cap T_{\bar{A}-u \mid}\right]}\right. \\
= & \sum_{u \in W_{U}}\left[\varrho_{+}^{\left|\left(D+\pi_{u}\right) \cap B\right|} \varrho_{-}^{\left|\left(D+\pi_{u}\right) \cap \bar{B}\right|}-\varrho_{+}^{|(D+u) \cap A|} \varrho_{-}^{|(D+u) \cap \bar{A}|}\right] \\
= & \delta\left[\sum_{|L| \text { even }} \varrho_{+}^{|D \backslash L|} \varrho_{-}^{|L|}-\sum_{|L| \text { odd }} \varrho_{+}^{|D \backslash L|} \varrho_{-}^{|L|}\right] \\
= & \delta \sum_{0 \leqq j \leqq|D|}(-1)^{j}\left(\begin{array}{c}
|D| \\
j
\end{array}\right) \varrho_{+}^{|D|-j} \varrho_{-}^{j}=\delta\left[\varrho_{+}-\varrho_{-}\right]^{|D|},
\end{aligned}
$$

as desired.

Let us conclude with the remark that we can find at least $n$ different toroidal sets $\alpha$ identical $\bar{\alpha}$, whenever some vertex of $\mathscr{W}_{U}$ has length $2^{n}$. Thus we have proved

Corollary 3.10. Let $U \in \mathfrak{U}^{-}$be any periodic interaction and $\mu \in \mathbb{R}$ some chemical potential. Then

either $|\operatorname{ex} \mathfrak{G}(\mu, U)|=\left|\operatorname{ex} \mathfrak{G}_{0}(\mu, U)\right|=1$

or $\quad|\operatorname{ex}(5)(\mu, U)| \geqq 2^{\left|\mathscr{W}_{U}\right|}$ and $\quad\left|\operatorname{ex} \mathfrak{G}_{0}(\mu, U)\right| \geqq\left|\mathscr{A}_{U}\right|$. 
Under certain circumstances the number of pairwise distinct pure phases, which are Spin Flip invariant and therefore all have the same particle density $\frac{1}{2}$, becomes arbitrarily large.

It would be interesting to know under what conditions the constructed measures $P^{M}$ and $P^{\alpha}$ are already all extremal and extremal invariant equilibrium states, respectively. E.g., consider the "periodic Ising interaction"

$$
\mathfrak{I}_{\beta, n}(t)=\left\{\begin{aligned}
-\beta & \text { if } t= \pm n_{j} e_{j} \quad(1 \leqq j \leqq v) \\
0 & \text { otherwise }
\end{aligned}\right.
$$

where $\beta>0, n=\left(n_{1}, \ldots, n_{v}\right) \in \mathbb{N}^{v}$ and $e_{j}$ the $j$-th unit vector $(1 \leqq j \leqq v)$.

Gallavotti's result [2] suggests that then in the two-dimensional case for sufficiently large $\beta$

and

$$
\mid \operatorname{ex}\left(\mathfrak{G}\left(\hat{\mu}\left(\mathfrak{J}_{\beta, n}\right), \mathfrak{J}_{\beta, n}\right) \mid=2^{n_{1} n_{2}}\right.
$$

$$
\mid \operatorname{ex}\left(\mathfrak{G}_{0}\left(\hat{\mu}\left(\mathfrak{J}_{\beta, n}\right), \mathfrak{J}_{\beta, n}\right)|=| \mathscr{A}_{\mathfrak{J}_{\beta, n}} \mid .\right.
$$

On the other hand, Dobrushin [6] has proved the existence of infinitely many (nonperiodic) extremal equilibrium states for the Ising interaction in three dimensions $(n=(1,1,1))$ for sufficiently large $\beta$. Thus

$$
\mid \operatorname{ex}\left(\mathfrak{G}\left(\hat{\mu}\left(\mathfrak{I}_{\beta, n}\right), \mathfrak{I}_{\beta, n}\right) \mid=\infty \quad \text { for large } \beta \text { and all } n .\right.
$$

Nevertheless, one could expect that

$$
\mid \operatorname{ex}\left(\mathfrak{G}_{0}\left(\hat{\mu}\left(\mathfrak{J}_{\beta, n}\right), \mathfrak{J}_{\beta, n}\right)|=| \mathscr{A}_{\mathfrak{I}_{\beta, n}} \mid \quad \text { for these } \beta \text { and } n .\right.
$$

\section{References}

1. Berge, C.: Principes de Combinatoire, Paris: Dunod 1968.

2. Gallavotti, G.: Commun. math. Phys. 27, 103-136 (1972).

3. Georgii,H.O.: Lecture Notes in Physics Vol. 16, Berlin-Heidelberg-New York: Springer 1972.

4. Meyer,P. A.: Probabilités et potentiel, Paris: Hermann 1966.

5. Pitt, H. R.: Proc. Cambridge Phil. Soc. 38, 325-343 (1942).

6. Dobrushin, R.L.: Teoriya Veroyatnostei i ee. Prim. 17, 619-639 (1972).

Hans-Otto Georgii

Institut $\mathrm{f}$. angewandte Mathematik

der Universität

D-6900 Heidelberg 1

Im Neuenheimer Feld 5

Federal Republic of Germany 